\title{
VELOCITY AUTO-CORRELATION FUNCTIONS IN A 2D LATTICE LORENTZ GAS: COMPARISON OF THEORY AND COMPUTER SIMULATION
}

\author{
D. FRENKEL ${ }^{1}$ \\ Physical Laboratory, State University Utrecht, P.O. Box 80.000, 3508 TA Utrecht, The Netherlands
}

Received 2 July 1986; revised manuscript received 26 February 1987; accepted for publication 17 March 1987

\begin{abstract}
We present Monte Carlo simulations of the hopping motion of a particle in a two-dimensional square lattice Lorentz gas. The long time tail of the velocity autocorrelation function in this system is computed with an accuracy of $1: 5 \times 10^{5}$. At low densities the Monte Carlo results agree quantitatively at all times with the predictions of kinetic theory. The theoretical predictions for the second order density corrections to the diffusion constant and the asymptotic behavior of the velocity autocorrelation function are found to be compatible with the Monte Carlo results. To our knowledge, this is the first example of quantitative agreement between kinetic theory and computer simulation for the long time tail of the velocity ACF in a Lorentz gas.
\end{abstract}

Modern kinetic theory was born with the discovery by Alder and Wainwright [1] of a long time tail in the velocity autocorrelation function (ACF) of hard spheres. Since then, long time tails have been observed in a wide variety of correlation functions. However, not all tails have the same origin [2]. Some are hydrodynamic in nature, such as the one observed by Alder and Wainwright. Hydrodynamic tails are at present reasonably well understood (see e.g. ref. [3]). Other tails (the so-called "molasses tail") appear to be associated with slow structural relaxation in dense fluids. A third type of long time tail is observed in the velocity ACF of a Lorentz gas. Unlike the previous two this tail is not due to collective motions, because in a Lorentz gas the moving particles only collide with static scatterers. Because of their relative simplicity, Lorentz gas models have been a favorite playground to compare kinetic theories with computer simulations [4-6]. The results of these comparisons have, thus far, been somewhat disappointing. The reason is that the kinetic theory predictions were valid only for low concentrations and long times. However, for the models studied, computer simulations could not be carried out with

\footnotetext{
1 Present address: FOM Institute for Atomic and Molecular Physics, P.O. Box 41883, 1009 DB Amsterdam, The Netherlands.
}

sufficient accuracy in this regime. To compare theory and simulations for higher concentrations, the effect of higher order density corrections must be estimated [7]. When this is done, there remains a discrepancy of a factor two between theory and the most accurate computer simulation data. This suggests either that the asymptotic regime has not been reached in the simulations, or that the theoretical description is not valid (or both).

In the present paper we compare kinetic theory and computer simulations for a particularly simple model, viz. a stochastic hopping model on a square lattice with excluded sites (hard scatterers). In this model, a test particle performs a random walk on the lattice. The waiting times between successive trial moves are Poisson distributed. At every move, the mobile test particle attempts to jump to one of four neighboring lattice sites, selected at random. If this lattice site is not excluded, the trial move is accepted, otherwise the test particle remains at its original location. This model is well suited for a comparison between kinetic theory and numerical simulation for the following reasons: first of all, the lowest order (linear) density expansion of the velocity ACF for this model is known analytically at all times [8], rather than just asymptotically. Secondly, the asymptotic behavior of the next order density correction to the velocity ACF is known [9]. And, last but not least, this model 
lends itself to simulation on a vector computer, which implies that we can compute the velocity ACF with a much higher accuracy, and therefore to longer times, than could be achieved in earlier simulations.

In order to test the range of validity of the theoretical expressions derived in ref. [8], we carried out Monte Carlo (MC) simulations on a square 2D lattice Lorentz gas, at low concentrations of the excluded sites. At these low concentrations (typically, $c=1.0-5.0 \%$ ) the expected amplitude of the $t^{-2}$ tail of the velocity ACF is small. E.g. after 100 attempted jumps, the absolute value of the velocity ACF should be of order $10^{-6}$. Clearly, in numerical simulations of long time tails, the statistical errors should be of this order of magnitude, or less. This seems difficult to achieve without excessively long calculations. The reason is the following: let us assume that we wish to compute the velocity ACF $\langle v(0) \cdot v(t)\rangle$ for $0<t<100$ with an accuracy of $1: 10^{6}$. Using Zwanzig and Ailawadi's estimate for the relative statistical error in a correlation function $C(t)[10]$,

$$
\begin{aligned}
& \left\langle\Delta C^{2}(t)\right\rangle / C^{2}(0) \approx \frac{4}{T} \int_{0}^{\infty} C^{2}(t) \mathrm{d} \tau / C^{2}(0) \\
& \approx 2 / N_{\text {samples }}
\end{aligned}
$$

we find that some $10^{12}$ independent samples of $v(0) \cdot v(t)$ for every value of $t$ are needed in order to achieve the desired accuracy. For 100 different $t$ values, this implies that such a calculation would require $10^{14}$ multiplications and additions just to compute the velocity ACF (i.e. excluding the actual Monte Carlo simulation). Assuming that the computation of the velocity $\mathrm{ACF}$ is a completely vectorizable point operation, it would take a computer like the Cyber 205 some 500 hours to carry out this task for every density.

We employed two tricks to reduce the amount of computing by more than two orders of magnitude. The first trick is based on the fact that we perform the actual simulation on a lattice Lorentz gas with discrete rather that continuous time steps. In the discrete Lorentz gas, trial moves are attempted at constant time intervals (i.e. the distribution of waiting times is a $\delta$-function, rather than a Poisson distribution). Choosing this time interval as our unit of time, and the spacing of the lattice as our unit of length, all particle velocities at a given time step are either 0 or \pm 1 . This enables us to represent all particle velocities by bit-vectors. The time consuming floating point multiplications are replaced by the much faster bit-vector operations. Once we have obtained the velocity ACF for the discrete-time lattice Lorentz gas, $\Phi_{\mathrm{d}}$, we can compute the continuous time ACF, $\boldsymbol{\Phi}_{\mathrm{c}}$, using the following relation, derived by Nieuwenhuizen et al. [11]:

$\Phi_{\mathrm{c}}(t)=\mathrm{e}^{-t} \sum_{s=0}^{\infty} \frac{t^{s}}{s !} \Phi_{\mathrm{d}}(s+1) \quad(t>0)$.

All the simulation results obtained for the discretetime model have been converted to the corresponding continuous-time expression using eq. (2). In principle, this procedure might entail a truncation error because one needs to know $\Phi_{d}$ at all times in order to compute $\Phi_{\mathrm{c}}$ with eq. (2). However, for the simulation results reported below, we only consider the behavior of $\Phi_{\mathrm{c}}$ for times that are about a factor of two shorter than the interval over which the $\Phi_{\mathrm{d}}$ 's were sampled. We checked that our computed $\Phi_{c}$ 's were insensitive to the precise asymptotic behavior of $\Phi_{\mathrm{d}}$ at times outside the interval sampled.

The second trick is designed to reduce the Zwanzig-Ailawadi error estimate (eq. (1)). To see how this is achieved, it is instructive to consider the velocity $A C F$ of a particle performing a random walk on a lattice without excluded sites. As successive steps in such an ideal random walk are uncorrelated, the velocity ACF reduces to a delta function at $t=0$. Next, consider exactly the same sequence of trial moves, but now with excluded sites present. As long as the test particle is not hitting an excluded site, the velocity is the same as in the previous case. But particle moves that would result in the particle occupying an excluded site are rejected. Hence, for those trial moves, the actual displacement and hence the "velocity" (defined as displacement per time step) is not equal to the velocity of the underlying ideal random walk. Let us denote the difference in velocity of a test particle for the same sequence of trial moves with and without excluded sites by $\delta v$. Clearly, the average magnitude of $\delta v$ is proportional to the density of such sites. Let us write the total velocity of a particle in the presence of hard obstacles as

$v_{\mathrm{tot}}(t)=v_{\mathrm{ran}}(t)+\delta v(t)$, 
where $v_{\text {ran }}(t)$ is the velocity of the ideal random walk. The expression for the velocity ACF now becomes

$$
\langle v(0) \cdot v(t)\rangle=\left\langle v(0) \cdot\left[v_{\operatorname{ran}}(t)+\delta v(t)\right]\right\rangle .
$$

As the random velocity $v_{\text {ran }}$ at time $t>0$ contains no memory of the previous history of the particle, it is not correlated to $v(0)$. Hence,

$$
\langle\boldsymbol{v}(0) \cdot v(t)\rangle=\langle v(0) \cdot \delta v(t)\rangle \quad(t>0) .
$$

The advantage of rewriting $\langle v(0) \cdot v(t)\rangle$ in this way is that the Zwanzig-Ailawadi estimate for the mean square error in the quantity on the right-hand side of eq. (5) is a factor $c$ lower than for the quantity on the right-hand side, where $c$ is the concentration of excluded sites.

By combing the two tricks described above, we were able to compute velocity ACFs with an accuracy of $(2-3) \times 10^{-6}$ in simulations that took about $1.5 \mathrm{~h}$ on a Cyber 205 computer. This accuracy is sufficient to determine the tail of the velocity ACF for times up to 100 mean collision times. As a test particle can move at most 100 lattice spacings in 100 time steps (and in the overwhelming majority of cases much less ), it was sufficient to study the diffusion process on relatively small $(128 \times 128)$ square attices. It should be stressed that finite size effects zan only show up at times long enough that a particle has a reasonable change to move over the linear dimensions of the box. In our case this would typically happen after some $10^{4}\left(=128^{2}\right)$ time steps.

We carried out simulations on square lattices containing $1,2.5,5,10$ and $15 \%$ hard scatters ${ }^{71}$. At the higher densities significant deviations from the (lowdensity) kinetic theory are to be expected. For every run we generated 500 different configurations of the square lattice with randomly distributed impurities. For every configuration we let 256 independent (i.e. noninteracting) test particles perform random walks during 12800 timesteps. All in all this amounts to 1.5 billion trial moves per simulation. The velocity ACF was accumulated during the run. As a check we compared the MC results for $\Phi_{\mathrm{d}}(s)$ for $s=1,2$ and 3 with the exact results which can easily be worked out. In all cases we found that the MC data reproduced the exact results to within the estimated error. Next, in

\# We used a Monte Carlo procedure similar to the one described by Pandey et al. [12]. order to facilitate comparison with the kinetic theory of ref. [8], we transformed the discrete time velocity $\mathrm{ACF}, \Phi_{\mathrm{d}}(s)$, to the corresponding continuous time expression, $\Phi_{c}$, using eq. (2)

Fig. 1 shows the tail of the velocity ACF, $\Phi_{c}$, for impurity concentrations between 1 and $5 \%$. The estimated error (obtained from the variance in the data for 500 independent configurations of scatterers) is indicated in the same figure. The drawn lines represent the predictions of kinetic theory, to linear order in the density. The agreement between theory and simulation is quite satisfactory, especially at short times. However, the discrepancy between the theoretical curve and the $\mathrm{MC}$ data is significant. The $\mathrm{MC}$ values for the tail of the velocity ACF appear to be systematically larger (in absolute value) than the low density kinetic theory expression. The difference is larger for the higher concentration results. This suggests that, even at these fairly low densities corrections to $\mathrm{O}\left(c^{2}\right)$ in the velocity ACF should be taken into account. If this is indeed the reason for the observed difference between the Monte Carlo data, $\Phi_{\mathrm{MC}}$, and $\Phi^{(1)}$, the $\mathrm{O}(c)$ kinetic theory expression, then the scaled difference $\Phi^{(2)}=\left(\Phi_{M C}-\Phi^{(1)}\right) / c^{2}$ should superimpose, at least for low densities. This comparison is shown in fig. 2 . As can be seen, the curves almost coincide for short times. At longer times the curves appear to go through a maximum, although, due to the statistical noise, its precise location is uncertain. The effect of this noise is worst at low concentrations. The figure suggests that the maximum is more pronounced at higher concentrations. This implies that, at these concentrations, third and higher order density corrections play a role. In fig. 2 we have also indicated the theoretical prediction for the second order density correction to the asymptotic tail of the velocity ACF. Clearly, the asymptotic regime is not yet reached after some 50 collision times. Moreover, the approach to the asymptotic value is not monotonic.

We can compare the theoretical predictions for the density-dependence of the diffusion constant $D(c)$ with the Monte Carlo results, writing

$D(c)=D^{(0)}+D^{(1)} c+D^{(2)} c^{2}+\ldots$.

For convenience, we have expressed $D(c)$ $\left(\equiv \int_{0}^{\infty} \mathrm{d} t \Phi(t)\right)$ in units of $D(c=0)$. We can estimate $D^{2}$ from our Monte Carlo data by determining the 

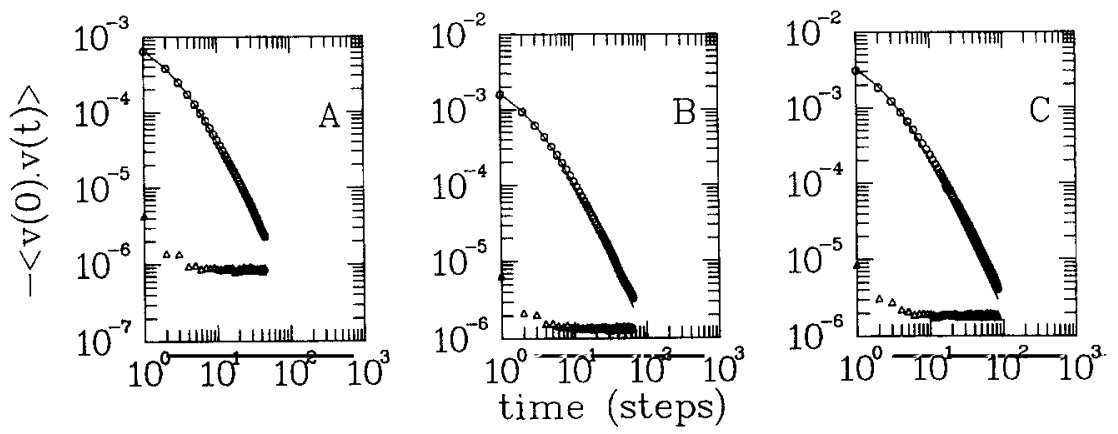

Fig. 1. The (negative) tail of the velocity autocorrelation function of the two-dimensional "lattice Lorentz gas" for $1 \%$ (A), $2.5 \%$ (B) and $5 \%$ (C) occupied sites. The Monte Carlo data points are denoted by open circles. The estimated errors in the Monte Carlo results are shown as open triangles. The drawn lines are the kinetic theory predictions of ref. [8] for the velocity ACF to lowest order in the density of scatterers. Note that only those simulation data points are shown that are at least a factor 2-3 above the statistical noise.

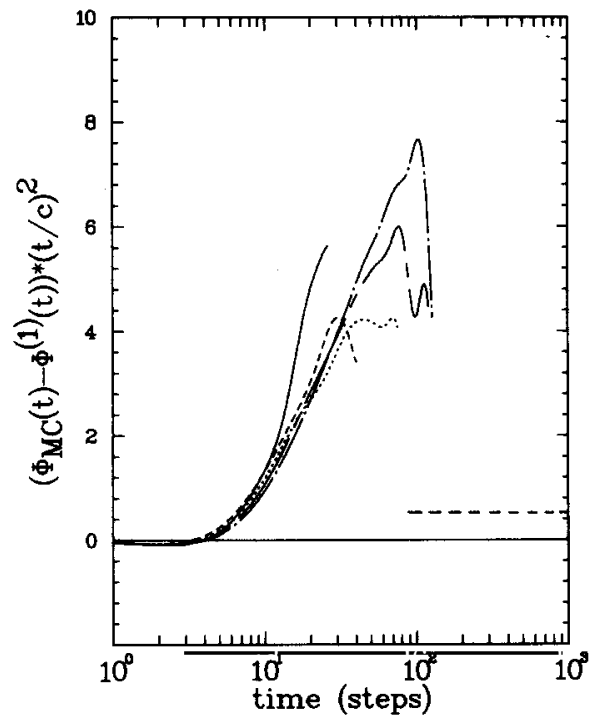

Fig. 2. Comparison between the kinetic theory predictions for the second order density correction to the asymptotic part of the tail of the velocity ACF of the two-dimensional "lattice Lorentz gas" [8] and the corresponding Monte Carlo data. In the figure, the difference between the full Monte Carlo velocity ACF and the lowest (linear) order kinetic theory prediction for this quantity has been multiplied by $t^{2}$ and divided by $c^{2}$. The dashed horizontal line corresponds to the second order density correction to the asymptote of the velocity ACF as given in ref. [8]. Simulation data: $1 \%$ scatterers $(-), 2.5 \%(---), 5 \%(-\ldots), 10 \%(--$ $--), 15 \%(-.-.-)$. At long times the simulation data are overwhelmed by noise, this problem is most serious for the lower concentrations. In the figure we do not plot the data beyond times where the estimated statistical error becomes $100 \%$.

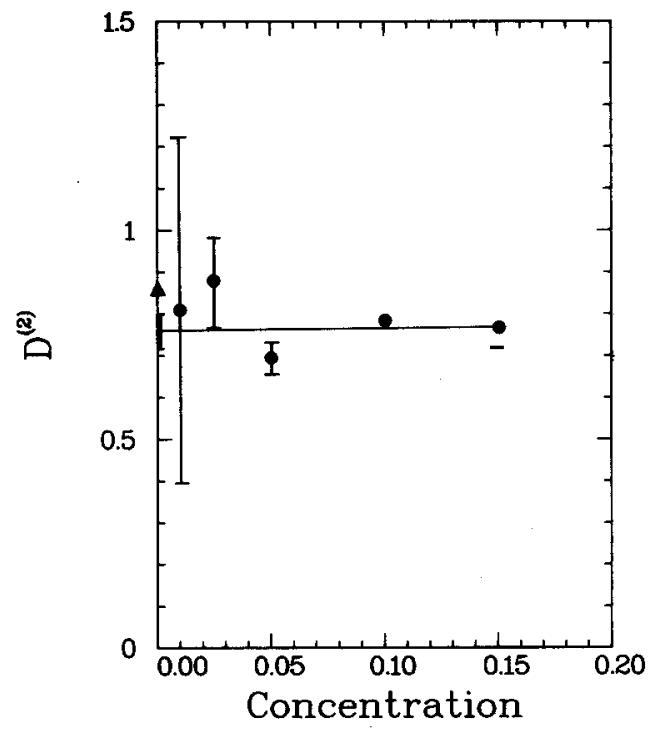

Fig. 3. Estimate of the second order density dependence of the diffusion constant. The points shown in the figure were computed from the Monte Carlo data using: $D^{(2)}=\left[D_{\mathrm{MC}}(c)-D^{(0)}-D^{(1)} c\right] / c^{2}$. The estimated errors (one standard deviation) are indicated in the figure, for the two highest densities the error bars are smaller than the dots. The drawn line is a least squares fit to the data points. The intercept of this line at $c=0\left(D^{(2)}=0.76 \pm 0.05\right)$ should be compared with the theoretical prediction $\left(D^{(2)}=0.85571\right.$, indicated by a filled triangle). Note that the theoretical value is within two standard deviations of the $\mathrm{MC}$ results.

zero-concentration intercept of $\left(D(c)-D^{(0)}-\right.$ $\left.D^{(1)} c\right) / c^{2}$. This comparison is shown in fig. 3. The estimated intercept is within two standard deviations of the theoretical value $D^{(2)}=0.85571$. The data 
are not sufficiently accurate to determine the sign (let alone the magnitude) of $D^{(3)}$.

It is important to note that knowledge of the full time dependence of the lowest order density expression for the velocity ACF has been crucial for the comparison between theory and simulation. If, in contrast, we had attempted to compare our Monte Carlo data directly with the presumed asymptote we might have been led to erroneous conclusions about the amplitude and/or exponent of the asymptotic tail. It is instructive to consider what would have happened if we had tried to "measure" the apparent amplitude or exponent of the tail of the velocity ACF from our MC data between 20 and 40 collision times. If we had made the (incorrect) assumption that after some 20 (attempted) jumps the velocity ACF decays with a simple power law then we would have been forced to conclude the following: Either the velocity ACF decays as $t^{-2}$, but the amplitude is larger than predicted by the linear density theory (for $1,2.5,5$, 10 and $15 \%$ hard scatterers these apparent ratios would have been: $1.24,1.33,1.46,1.81$ and 2.09 respectively). Or, if we had not assumed a $t^{-2}$ decay, we would have found a $t^{-\alpha}$ decay, with a non-universal value of $\alpha$. For the same concentrations of excluded sites as above, a least-squares fit yields the following values for $\alpha$ : $\alpha=2.05(1 \%), 1.93(2.5 \%)$, $1.82(5 \%), 1.68(10 \%)$ and $1.52(15 \%)$. It should be stressed that the fact that the lattice Lorentz gas has not yet reached asymptotic behavior after 20 jumps does not imply that the same holds for continuous 2D Lorentz gas after 20 collisions. The reason is that in a continuous Lorentz gas [6] 20 impermeable scatterers are visited in 20 collisions. For the dilute lattice model only a few hard scatteres are visited in this number of jumps.

The simulations reported in this paper were carried out following many stimulating discussions with Matthieu Ernst, Theo Nieuwenhuizen and Peter van Velthoven. I gratefully acknowledge their continuous interest and support. Computer time on the Amsterdam Cyber 205 was kindly made available by the University of Utrecht and Control Data Corporation.

\section{References}

[1] B.J. Alder and T.W. Wainwright, Phys. Rev. A 1 (1970) 18.

[2] B.J. Alder in: Proc. 97th Int. School of Physics "Enrico Fermi" (North-Holland, Amsterdam), to be published.

[3] J. Erpenbeck and W.W. Wood, Phys. Rev. A 32 (1985) 412.

[4] C. Bruin, Phys. Rev. Lett. 29 (1972) 1670.

[5] J.C. Lewis and J.A. Tjon, Phys. Lett. A 66 (1978) 349.

[6] B.J. Alder and W.E. Alley, J. Stat. Phys. 19 (1978) 341; Physica A 121 (1983) 523.

[7] M.H. Ernst, J. Machta, J.R. Dorfman and H. van Beijeren, J. Stat. Phys. 34 (1984) 477; 35 (1984) 413.

[8] Th. M. Nieuwenhuizen, P.F.J. van Velthoven and M.H. Ernst, Phys. Rev. Lett. 57 (1986) 2477.

[9] P.J.H. Denteneer and M.H. Ernst, Phys. Rev. B 29 (1984) 1755;

Th.M. Nieuwenhuizen and M.H. Ernst, J. Stat. Phys. 41 (1985) 773.

[10] R. Zwanzig and N.K. Ailawadi, Phys. Rev. 182 (1969) 280.

[11] Th.M. Nieuwenhuizen, P.F.J. van Velthoven and M.H. Ernst, submitted for publication.

[12] R.B. Pandey, D. Stauffer, A. Margolina and J.G. Zabolitzky, J. Stat. Phys. 34 (1984) 427. 